\title{
IDENTIFICACIÓN DE CONGLOMERADOS PARA IMPULSAR LAS CADENAS PRODUCTIVAS DE CARNE EN MÉXICO ${ }^{1}$
}

\author{
Gabriela Rodríguez-Licea², José Alberto García-Salazar³, Juvencio Hernández-Martínez ${ }^{4}$
}

\begin{abstract}
RESUMEN
Identificación de conglomerados para impulsar las cadenas productivas de carne en México. El objetivo del presente trabajo fue realizar un análisis de correlación espacial regional de la producción de carne en canal por especie en México. El estudio se llevó a cabo durante el periodo 2000 -2012. A través del análisis exploratorio de datos, de la estimación del índice de Moran y de la prueba de LISA, se buscó evidencias de dependencia espacial e identificar conglomerados que impulsen la integración de las cadenas productivas. El análisis exploratorio aporta evidencia de que los estados del norte pueden formar conglomerados que impulsen la producción carne de bovino, porcino y pollo; y el incremento en la producción de carne de ovino y caprino, podría darse entre estados de la región centro y del norte, respectivamente. Los índices positivos obtenidos para la carne de bovino, ovino y caprino, mostraron que la vecindad de estados productores no afectaría la conformación de conglomerados para incrementar la producción; mientras que, la autocorrelación negativa para la carne de porcino y pollo, hace suponer que la situación de vecindad de un estado productor se vería afectada por los agrupamientos regionales de sus vecinos. Con la prueba de LISA, se identificaron grupos estadísticamente significativos que soportan la evidencia de que la concentración espacial de la producción favorece la conformación de clúster y la integración de cadenas productivas únicamente para la carne de bovino, ovino y caprino.
\end{abstract}

Palabras clave: autocorrelación espacial, análisis exploratorio de datos, producción regional, índice de Morán, prueba de LISA.

\begin{abstract}
Identification of clusters to boost the productive chains of meat in Mexico. The aim of this study was to analyze the regional spatial correlation of meat carcass production by species in Mexico. The study was carried out between 2000-2012. Through exploratory data analysis, the estimation of the Moran Index and LISA test, the study tried to find evidence of spatial dependence and to identify clusters that promote the integration of productive chains. The exploratory analysis provided evidence that the northern states can generate clusters to boost production of beef, pork, and chicken; and that the increase in the production of sheep and goat meat may occur in the central and northern states, respectively. The positive indexes for beef, sheep, and goat meat showed that the neighborhood between producer states would not affect the creation of clusters to increase production; while the negative autocorrelation for pork and chicken suggests that the neighborhood of a producer state would be affected by its neighbors' regional clusters. LISA test allowed to identify statistically significant groups that support the evidence that spatial concentration of production benefits the creation of clusters and the integration of productive chains only for beef, sheep, and goat meat.
\end{abstract}

Keywords: spatial autocorrelation, exploratory data analysis, regional production, Moran's Index, LISA test.

\footnotetext{
1 Recibido: 19 de mayo, 2015. Aceptado: 12 de agosto, 2015. Este trabajó fue el resultado de talleres tomados de la Facultad de Economía de la Universidad Autónoma del Estado de México sobre econometría espacial y el manejo del software Open Geoda, México.

2 Universidad Autónoma del Estado de México. Cerro de Coatepec S/N, Ciudad Universitaria. Toluca México, Apdo. Postal 50110. gabyr11972@hotmail.com

3 Colegio de Posgraduados en Ciencias Agrícolas. Carretera México-Texcoco, km 36.5, Montecillo, Texcoco Apdo. postal 56230, Estado de México.jsalazar@colpos.mx

4 Universidad Autónoma del Estado de México. Centro Universitario UAEM-Texcoco. Av. Jardín Zumpango, s/n Fracc. El Tejocote, Texcoco, Edo. de México, México C.P. 56259. Teléfono: (01 595) 92103 68.jh_martinez1412@yahoo.com.mx
} 


\section{INTRODUCCIÓN}

La carne constituye uno de los componentes más importantes en la alimentación y nutrición de la población; de ahí que en México su producción sea una de las actividades más importantes dentro del sector primario, al aportar el 32,31\% del producto interno bruto agropecuario anual (PIBAA) (CNOG, 2013). No obstante, la integración de las cadenas productivas ha causado la incubación de nichos de mercado que propician déficit o superávit a nivel estatal, dada la concentración espacial y especialización de la producción, y la distancia que existe de los centros de producción a los de consumo; situación que ha favorecido la producción de carne de algunas especies y la participación en la producción nacional de ciertos estados.

De las 5,22 millones de toneladas (MDT) producidas de carne en canal al año durante el período 2000-2012, el 98,29\% correspondió a carne de pollo, bovino y porcino, generando el sector ovino-caprino solamente el $1,71 \%$ de la producción nacional. Aunado a lo anterior, el grado de integración de las cadenas productivas propició que el $45,88 \%$ de la producción se concentrara espacialmente en seis estados, sobresaliendo Jalisco en la carne de porcino, pollo y bovino; Veracruz en bovino, pollo y ovino; Sonora y Guanajuato en cerdo, Puebla en caprino y Durango en pollo (SIACON, 2013).

Chiapas, Coahuila, Oaxaca, el Estado de México e Hidalgo, se han destacado en la producción nacional; sin embargo, su nivel de especialización en la producción, una menor accesibilidad a gramíneas, granos y pastas de oleaginosas, la poca disponibilidad de infraestructura para el sacrificio; y su lejanía con respecto a importantes centros de consumo, no les ha permitido una mayor integración de sus cadenas productivas.

En los últimos años los estudios sobre economía regional han venido desarrollando herramientas y métodos de análisis espacial que permiten evaluar la existencia de relaciones socioeconómicas entre agentes y, a la vez, la relación de estos con su ubicación geográfica. La utilización de este tipo de herramientas tiene la ventaja de permitir, para el caso particular de este estudio, establecer si la producción de carne por especie en los estados se distribuye en forma aleatoria o, si por el contrario, existe evidencia de algún tipo de dependencia espacial.
Con el fin de identificar las entidades que pudieran integrar conglomerados, para la integración de sus cadenas productivas, partiendo de la hipótesis de que existe un efecto generado por la vecindad espacial entre los estados productores de carne por especie que pueden o no favorecer la conformación de conglomerados entre entidades, se planteó como objetivo del presente trabajo realizar un análisis de correlación espacial regional de la producción de carne en canal por especie en México.

\section{MATERIALES Y MÉTODOS}

De acuerdo con Anselin (1998), la econometría espacial es una herramienta que permite establecer la relación funcional entre un punto dado en el espacio y lo que ocurra en cualquier otro punto, e identificar si existe o no dependencia espacial para la formación de conglomerados. Dado $\mathrm{y}_{\mathrm{i}}=\mathrm{f}\left(\mathrm{y}_{\mathrm{i}}\right)$; e $\mathrm{i}=1,2,3, \ldots, \mathrm{n}$, con $\mathrm{j} \neq \mathrm{i}$; la expresión matemática queda expresada como:

$$
\operatorname{Cov}\left[y_{i} y_{j}\right]=E\left[y_{i} y_{k}\right]-E\left[y_{i}\right]-E\left[y_{j}\right] \neq 0 \text { para } i \neq j
$$

La expresión (1) indica que si una observación asociada a una localización (i) se relaciona con otra observación en una localización j jæi, tal relación se expresa por el momento condicional de la covarianza entre ambas localizaciones. Partiendo de lo anterior, para establecer la existencia de patrones de comportamiento en los datos utilizados, sin tener en cuenta factores espaciales, se realizó el análisis exploratorio de datos; y, a través del Exploratory Spatial Data Analysis se estableció la existencia de características espaciales en los indicadores de producción, mediante la identificación de conglomerados. Adicionalmente, se efectuó un análisis a través de indicadores de asociación espacial local, el cual permitió evaluar la aleatoriedad espacial, comparando los valores del indicador en cada estado con los de los estados vecinos. Finalmente, por medio de la estimación del índice de Morán y su gráfico de dispersión, fue posible descomponer el tipo de asociación espacial, cada una de las cuales correspondió a uno de los cuadrantes del plano cartesiano. 


\section{Autocorrelación espacial}

Uno de los supuestos básicos de los modelos econométricos es que no deben presentar problemas de autocorrelación serial, la cual de acuerdo con Gujarati (1995), plantea la correlación de rezagos de una serie dada consigo misma en un número de unidades de tiempo. De acuerdo con este autor, al trasladar este concepto a datos transversales con algún criterio geográfico, los términos del error podrán estar relacionados entre sí y, por lo tanto, presentar autocorrelación espacial.

De acuerdo con Anselin (1998), la autocorrelación espacial es positiva cuando un fenómeno determinado en una región dada, propicia su expansión a otras regiones circundantes y dicha expansión genera la concentración del mismo; y, es negativa, cuando ciertos fenómenos en una región impiden u obstaculizan la aparición de estos en otras regiones vecinas. Las causas de autocorrelación espacial puede ser: autocorrelación residual (derivados de la posibilidad de escasa correspondencia entre el ámbito espacial del fenómeno de estudio y las unidades espaciales de observación, los errores de medición serán muy probables) y la autocorrelación sustantiva (lo que ocurre en un territorio afectó a otros territorios, lo que ocurre en un punto en el espacio está determinado por lo que pasa en otro u otros puntos, debido a interdependencias en tiempo y espacio en unidades espaciales).

Los fenómenos que causan la autocorelación espacial deben ser incorporados dentro de los modelos econométricos y estadísticos para detectar su presencia, dado que esta se vincula con variables no homogéneas agrupadas de formas muy diversas en el espacio, y plantea relaciones multidireccionales, donde cada observación posee distintos tamaños, ubicaciones, distancias entre ellas, etc. Un instrumento a través del cual se puede expresar la dependencia espacial y resolver el problema de multidireccionalidad en la modelación de este efecto espacial, es la matriz de pesos espaciales.

\section{Matriz de pesos espaciales}

La matriz de pesos espaciales son instrumentos que fusionan la interdependencia y las relaciones multidireccionales, se definen por la letra $\mathrm{W}$ (por la palabra inglesa weight, peso) y, matemáticamente, se representa de la siguiente forma:

$$
W=\left[\begin{array}{cccc}
0 & W_{12} & \cdot & W_{1 N} \\
W_{21} & 0 & \cdot & W_{2 N} \\
\cdot & \cdot & \cdot & \cdot \\
W_{N 1} & W_{N 2} & \cdot & 0
\end{array}\right]
$$

Donde, $\mathrm{w}_{\mathrm{ij}}$ son los coeficientes de la matriz W, cuyos valores binarios serán igual a 0 si las regiones i y j no son vecinas y 1 si lo son. Dado que la matriz es simétrica, transpuesta y, por lo tanto cuadrada, sus elementos $\mathrm{w}_{\mathrm{ij}}$ representan la interdependencia existente entre las regiones i y j y serán estocásticos o exógenos al modelo.

\section{Estadístico de dependencia espacial: índice de Morán}

Un instrumento para contrastar la correlación espacial dentro del análisis exploratorio es el índice de Morán (I) (1948), a través del cual se puede incorporar la matriz de pesos espaciales $(W)$. Matemáticamente, el índice se puede expresar como:

$$
I=\frac{R}{\sum_{i} \sum_{j} w_{i j}} \frac{\sum_{i} \sum_{j} w_{i j}\left(x_{i}-\bar{x}\right)(x j-\bar{x})}{\sum_{i}\left(x_{i}-\bar{x}\right)^{2}}
$$

Donde $\mathrm{X}_{\mathrm{i}}$ es la variable cuantitativa en el estado $i$, es su media muestral, $\mathrm{w}_{i j}$ son los pesos de la matriz W (matriz de pesos espaciales) y $\mathrm{R}$ es el tamaño de la muestra (estados). Bajo la hipótesis nula de autocorrelación, el estadístico de Morán es asintóticamente normal.

De acuerdo con Moreno y Vayá (2000), el I sigue una distribución normal estandarizada en muestras grandes, de tal forma que un valor positivo (negativo) significativo del índice Z(I) llevará al rechazo de la hipótesis nula de no autocorrelación espacial y a la aceptación de autocorrelación espacial positiva (negativa). La información del índice se puede graficar 
en un diagrama de dispersión de Morán, el cual presentó en el eje horizontal a la variable $x$ normalizada y en el eje vertical a la variable multiplicada por la matriz de pesos $\mathrm{W}$, lo cual da lugar al retardo espacial de dicha variable.

A través del cálculo del I y su gráfico de dispersión, se pudo descomponer el tipo de asociación espacial en cuatro categorías, cada una de las cuales correspondiente a cada uno de los cuadrantes del plano cartesiano. En el cuadrante I se colocaron los estados con alta producción de carne y que estaban rodeados de estados vecinos que también generaban una alta producción. En el cuadrante III, por el contrario, se ubicaron los estados con baja producción que estaban rodeados por estados poco productivos. En contraste con los cuadrantes I y III, en el cuadrante II se encontraron los estados con producción baja pero rodeados de estados con alta producción; y, en el cuadrante IV, se ubicaron aquellos estados con alta producción pero rodeados por vecinos poco productivos.

Si los puntos se encuentran en los cuadrantes I y III, el estadístico de Morán tomará un valor positivo, mientras que si los puntos se ubican en los cuadrantes II y IV, el valor será negativo; a partir de esto, la asociación espacial puede ocurrir de la siguiente manera: cuadrante 1 , alto en $\mathrm{X}$ y alto en $\mathrm{Y}(+,+)$; cuadrante II, bajo en $\mathrm{X}$ y alto en $\mathrm{Y}(-,+)$; cuadrante III, bajo en $\mathrm{X}$ y bajo en $\mathrm{Y}(-,-)$; y cuadrante IV, alto en $\mathrm{X}$ y bajo en $\mathrm{Y}(+,-)$ (Figura 1$)$.

La condición multidireccional de la dependencia espacial: una región puede estar afectada no solamente por otra región contigua o vecina, sino por otras que la rodean, al igual que ella puede afectar a las otras; imposibilitando la utilización del operador de rezago $\mathrm{L}, \mathrm{L}^{\mathrm{p}} \mathrm{Y}=\mathrm{Y}_{\mathrm{t}-\mathrm{p}}$, presente en el contexto temporal, por lo que para el análisis de dependencia espacial se utilizó la matriz $\mathrm{W}$ de efectos espaciales como operador de rezago espacial, la cual es una media ponderada de los valores vecinos y, matemáticamente se puede expresar como:

$$
W Y=\sum_{j=1}^{N} w_{i j} y_{j}
$$

Donde $y_{j}$ es el valor que toma el atributo medido en la vecindad $\mathrm{j}, \mathrm{y}_{\mathrm{ij}}$ es un ponderador cuya suma es la unidad.

La dependencia espacial implicaría que, al tomar en consideración una variable económica para

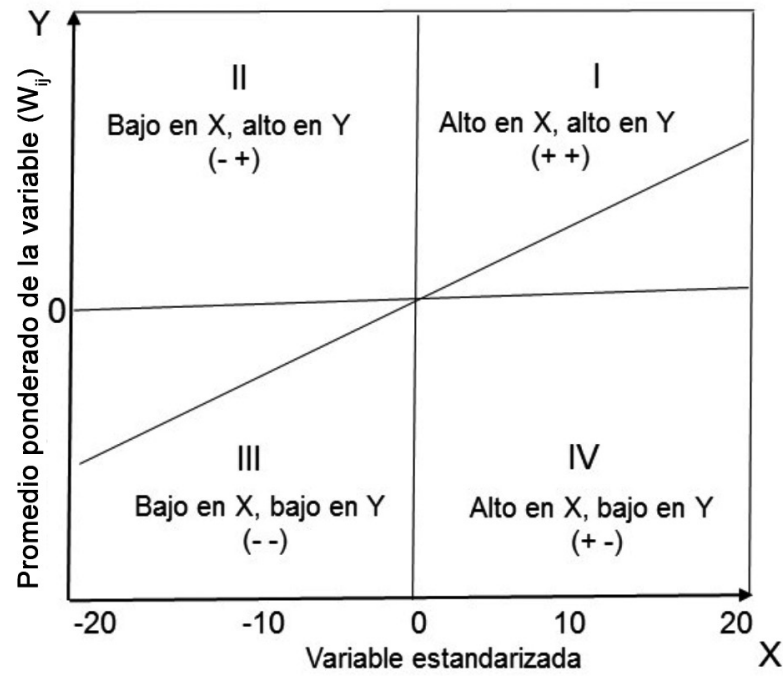

Figura 1. Diagrama de dispersión del índice de Morán de los cuatro cuadrantes para la producción de carne a nivel estatal, 2000-2012. México.

Figure 1. Dispersion diagram for Moran's Index from four quadrants of meat production at state level 20002012. Mexico.

diferentes localidades, se tendrían más características similares en localidades vecinas que en aquellas separadas por grandes distancias: de ser la positiva la dependencia, la presencia de un atributo en una localidad se extendería a las regiones vecinas; en caso contrario, obstaculizaría su presencia en sus vecindades (Quintana y Mendoza, 2008); es decir, que la concentración de la producción de carne de determinada especie depende de las bondades y estructura productiva de sus vecinos regionales, lo que supondría la conformación de regiones.

\section{Indicador local de asociación espacial (LISA)}

En procesos donde existen patrones de agrupación local o conglomerados, el I no los puede detectar, por lo que, de acuerdo a Anselin (1995), una alternativa para identificar las agrupaciones locales es el índice local de Morán, calculado en cada estado y, definido matemáticamente como:

$$
I_{i}=\frac{z_{i}}{\sum_{i} z_{i}^{2} / N_{j}} \sum_{i} w_{i j} z_{j}
$$


Donde $\mathrm{I}_{\mathrm{i}}$ es el índice local, $\mathrm{z}_{\mathrm{i}}$ el valor de la variable correspondiente al estado $i, \mathrm{~N}_{\mathrm{j}}$ el conjunto de estados vecinos a $i$. Un valor elevado positivo (negativo) y significativamente estadístico, da lugar a la existencia de un conglomerado alrededor del estado $i$ de valores similares elevados (bajos). Con base en $\mathrm{I}_{\mathrm{i}}$, es posible encontrar su contribución al índice global de I, y detectar sus valores extremos, lo cual lo convierte en un LISA.

\section{Variables utilizadas y fuentes de información}

El análisis, se derivó del volumen acumulado de la producción de carne en canal por especie animal, obtenido del reporte de los boletines de información económica pecuaria de la Confederación Nacional de Organizaciones Ganaderas (CNOG, 2013). Las estimaciones se realizaron con el software OpenGeoDa ver. 0.9.8.14.

\section{RESULTADOS}

\section{Análisis exploratorio de los datos}

El análisis exploratorio permitió identificar el comportamiento de la producción de carne por especie y mapear su distribución geográfica, la cual sirvió de base para identificar la participación estatal en la producción nacional durante 2000-2012.

Haciendo referencia al comportamiento de la producción por especie, de manera general, durante el período de análisis se generó una producción promedio anual de 5,22 MDT con crecimiento del 2,67\%; de esta, el 45,96\% fue aportado por el sector avícola, ocupando el segundo lugar la carne de bovino con el $30,85 \%$, y el tercero la de porcino con el $21,48 \%$. Por su parte, la carne de ovino solo representó el 0,90\% del total nacional, a pesar de haber registrado el mayor crecimiento en la producción, con una tasa promedio anual de 4,66\%; esto contrasta con lo observado para la carne de caprino, la cual únicamente creció en $0,57 \%$ al año, con una participación en la producción total del $0,81 \%$.

El mapeo de la distribución geográfica mostró un alto grado de concentración de la producción a nivel estatal, dado que el $45,88 \%$ se concentró en seis entidades, las cuales hicieron la siguiente aportación a la producción nacional: Jalisco, 12,73\%; Veracruz, 10,95\%;
Sonora, 5,68\%; Guanajuato, 5,67\%; Puebla, 5,61\%; y Durango, $5,23 \%$. Aunado a lo anterior, el mapeo evidenció que existe un alto grado de concentración de la producción a nivel estatal por especie; sobresaliendo en la carne de bovino Veracruz, Jalisco y Chiapas con una aportación conjunta del $32,05 \%$, y una individual de $14,31,11,37$ y $6,31 \%$, respectivamente.

Veracruz, Jalisco y Chiapas, mostraron un alto grado de integración de la cadena productiva, derivado de la especialización en la producción, la disponibilidad y aprovechamiento de gramíneas, forrajes, granos y pastas de oleaginosas para la alimentación del ganado o para la elaboración de alimento balanceado; así como, de infraestructura para el sacrificio de ganado, y la cercanía con grandes centros de consumo. Particularmente, Jalisco ocupó el primer lugar en la producción de forrajes con una aportación promedio anual de 12,38\% del total nacional, y en la de alimento balanceado con el $17,59 \%$ del obtenido en las industrias mixtas y en las plantas integradas verticalmente (ANFACA, 2013); además, de los rastros municipales y tipo inspección federal (TIF) disponibles a nivel nacional para el sacrificio de ganado bovino, el 10,33\% se encontraban en esta entidad (SENASICA, 2012).

Los estados de la frontera norte (Baja California, Chihuahua, Durango, Sinaloa y Sonora) no fueron los más importantes de manera individual en la producción; no obstante, su ubicación geográfica pudo favorecer la formación de conglomerados en esa región, dado que fue el segundo grupo más importante en la producción de este tipo de carne con una aportación conjunta del 22,69\% (Figura 2). Su formación reforzaría la comercialización de ganado para la obtención de carne realizada por Sonora, dado que comercializó animales en pie a Baja California, Sinaloa y Chihuahua para la engorda; y vacas en pie a Sinaloa, Baja California y Chihuahua. Por otro lado, seguiría impulsando el crecimiento promedio anual de la producción de carne en Sinaloa equivalente al $5,20 \%$, y coadyuvaría a que Durango incremente su producción, ya que es la única entidad de este grupo que registró tasas de crecimiento negativas a lo largo del período.

Con relación a la carne de porcino, se encontró evidencia de la fuerte concentración estatal de la producción: Sonora y Jalisco aportaron conjuntamente cerca del $40 \%$ de la producción nacional, Guanajuato, Puebla y Yucatán alrededor del 25\%; y Veracruz 


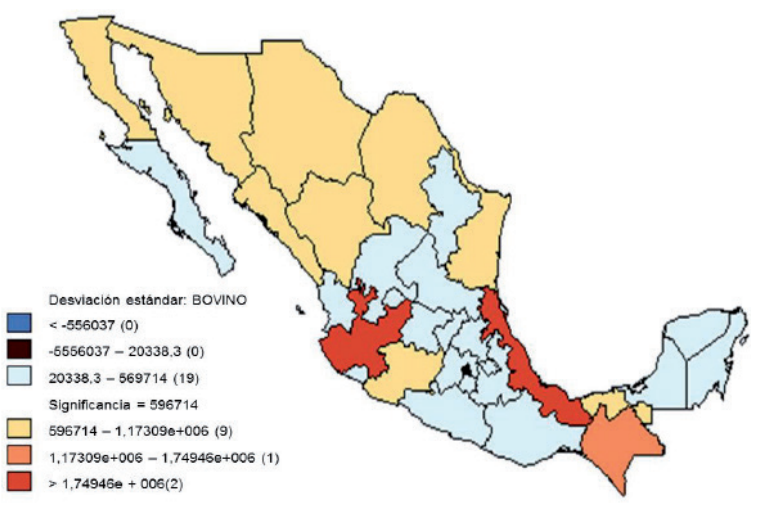

Figura 2. Participación estatal y mapeo de la distribución geográfica de la producción de carne en canal de bovino 2000-2012, México.

Fuente: elaboración propia con OpenGeoDa ver. 0.9.8.14, a partir de datos de la CNOG (2013).

Figure 2. State participation and mapping of the geographical distribution of cattle carcass production 20002012, Mexico.

Source: own elaboration with OpenGeoDa view 0.9.8.14, using data from the CNOG (2013).

y Michoacán un poco más del 10\% (Figura 3). El conglomerado de cerdos articulado con el de granjas productoras de cabezas, con el de alimentos y con el sector agrícola de la región del sur de Sonora, posicionó a esa entidad en primer lugar a nivel nacional, ya que contó con un inventario de 1572397 cabezas producidas en 354 granjas, y una infraestructura para el sacrificio equivalente al 20,43\% del total nacional.

Colima y Aguascalientes no aportaron ni el uno por ciento de la producción nacional, no obstante, registraron los mayores crecimientos en la producción con una tasa promedio anual de 14,66 y 9,83\%, respectivamente, lo que significa que, dada su localización geográfica, en un futuro podrían integrar conglomerado con los estados del occidente.

En el caso de la carne de pollo, los principales productores fueron Jalisco, Veracruz, Durango, Querétaro, Aguascalientes, Puebla y Guanajuato, con una aportación conjunta de 58,74\% (Figura 4). La elevada agregación espacial fue resultado de la concentración de la producción por sistema productivo y a nivel empresarial, dado que el $70 \%$ de esta se generó en granjas tecnificadas, $20 \%$ en semitecnificadas y $10 \%$ en traspatio; situación que contribuyó a que

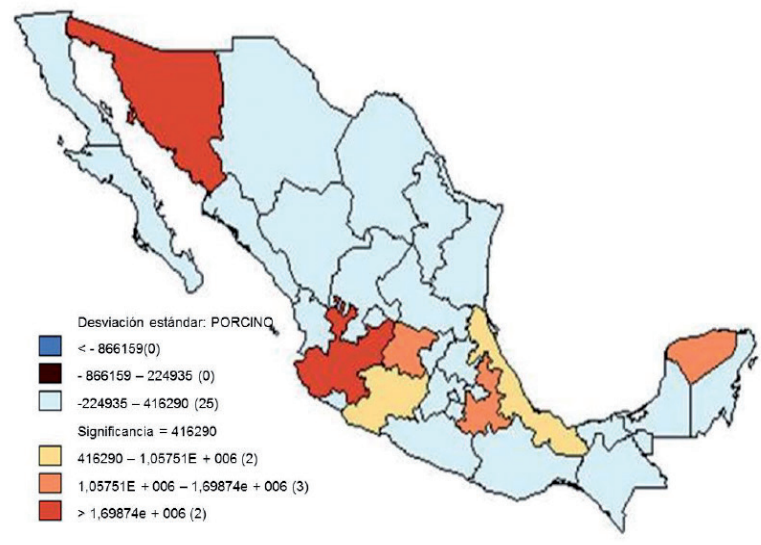

Figura 3. Participación estatal y mapeo de la distribución geográfica de la producción de carne en canal de porcino 2000-2012, México.

Fuente: elaboración propia con OpenGeoDa ver. 0.9.8.14, a partir de datos de la CNOG (2013).

Figure 3. State participation and mapping of the geographical distribution of pork carcass production 2000-2012, Mexico.

Source: own elaboration with OpenGeoDa view. 0.9.8.14, using data from the CNOG (2013).

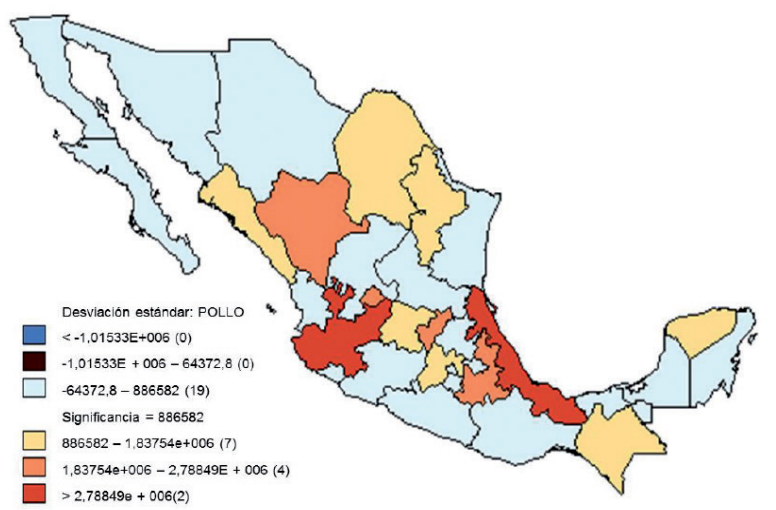

Figura 4. Participación estatal y mapeo de la distribución geográfica de la producción de carne en canal de pollo 2000-2012, México.

Fuente: elaboración propia con OpenGeoDa ver. 0.9.8.14, a partir de datos de la CNOG (2013).

Figure 4. State participation and mapping of the geographical distribution of chicken carcass production 20002012, Mexico.

Source: own elaboration with OpenGeoDa view 0.9.8.14, using data from the CNOG (2013). 
el $80 \%$ se aglutinará en tres empresas: Bachoco, Pilgrim's y Tyson, las cuales al contar con sistemas altamente tecnificados adaptados a las condiciones del mercado del país, lograron cubrir de manera directa $54 \%$ de la demanda nacional, representada por mercados minoristas de los principales centros urbanos, con productos procesados obtenidos en rastros TIF destinados al consumo directo.

El resto del mercado fue cubierto por cerca de 200 pequeños y medianos productores, ubicados en entidades con menor participación como Chiapas, Estado de México, Nuevo León, Sinaloa y Yucatán; los cuales, a pesar de la alta vulnerabilidad ante cambios de los precios y la demanda, lograron cubrir mercados regionales en expansión, debido a que su localización espacial los limita a formar cluster que los coadyuven a incrementar su cobertura de mercado. No obstante, Chiapas y Sinaloa incrementaron su producción en 9,12 y $5,1 \%$, respectivamente.

En cuanto a la carne de ovino, el Estado de México, Hidalgo y Veracruz generaron el mayor volumen de la producción con una aportación conjunta del 28\% del total nacional, ocuparon el segundo lugar grupal Puebla, Zacatecas, San Luis Potosí y Sinaloa con una participación del 20,92\% (Figura 5). A pesar

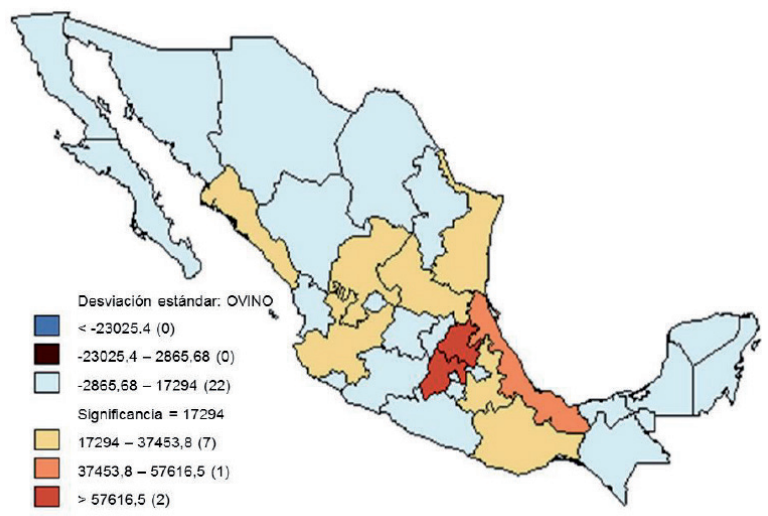

Figura 5. Participación estatal y mapeo de la distribución geográfica de la producción de carne en canal de ovino, 2000-2012, México.

Fuente: elaboración propia con OpenGeoDa ver. 0.9.8.14, a partir de datos de la CNOG (2013).

Figure 5. State participation and mapping of the geographical distribution of sheep carcass production 20002012, Mexico.

Source: own elaboration with OpenGeoDa view 0.9.8.14, using data from the CNOG (2013). de que el Estado de México ocupó el primer lugar nacional, su producción no cubrió su demanda interna mensual, la cual superó sesenta mil canales, por lo que tuvo que adquirir animales en pie para su sacrificio y carne de Querétaro, Guanajuato, Jalisco, San Luis Potosí, Zacatecas, Veracruz, Chihuahua y Coahuila. Similarmente, la transición de las unidades semiempresariales de producción ovina de Hidalgo, han dificultado cubrir la demanda estatal y del mercado, esto a pesar de haber registrado crecimientos anuales en la producción de $1,56 \%$.

En contraste con el Estado de México e Hidalgo, la diversidad climatológica de Veracruz ha favorecido la implementación de diversos sistemas productivos que generaron en promedio al año $4775 \mathrm{t}$ de carne, distribuidas en el sur, centro y norte, con una participación regional estatal de $41,8,35,5$ y $22,7 \%$, respectivamente; también su ubicación geográfica y la poca variación estacional en la oferta, le ha permitido cubrir importantes mercados de los principales estados consumidores de este producto, tales como el Distrito, Estado de México, Puebla, Hidalgo, Querétaro y Morelos, entidades que demandaron cerca del $85 \%$ de la carne de ovino, de la cual el $90 \%$ se consume en forma de barbacoa (Partida de la Peña et al., 2013).

Las principales entidades productoras deberán incrementar el grado de integración de sus cadenas productivas, a fin de evitar que en el futuro el segundo grupo productivo más importante del país, o estados como Campeche, Chihuahua, Jalisco o Sonora las desplacen en la participación nacional, dado que, durante el período de análisis, estos últimos incrementaron su producción anual en 14,97, 14,55, 11,86 y $11,14 \%$, respectivamente.

En la producción de carne de caprino, Coahuila, Oaxaca, Puebla, Guerrero, Zacatecas y San Luis Potosí, sobresalieron como principales productores, al generar conjuntamente $51,9 \%$ de la producción nacional, aportando los dos primeros más de la quinta parte de esta (Figura 6), lo que dejó ver que más de la mitad de la producción se concentró en estados del norte y sur (excepto Puebla), aunque entidades del occidente destacadas por su participación en la producción de carne de bovino, porcino y pollo, como Michoacán, Jalisco y Guanajuato, han incrementado su producción en 5,84, 5,28 y 4,59\%, respectivamente, lo cual podría favorecer la formación de clusters que conlleven a incrementar su participación en la 


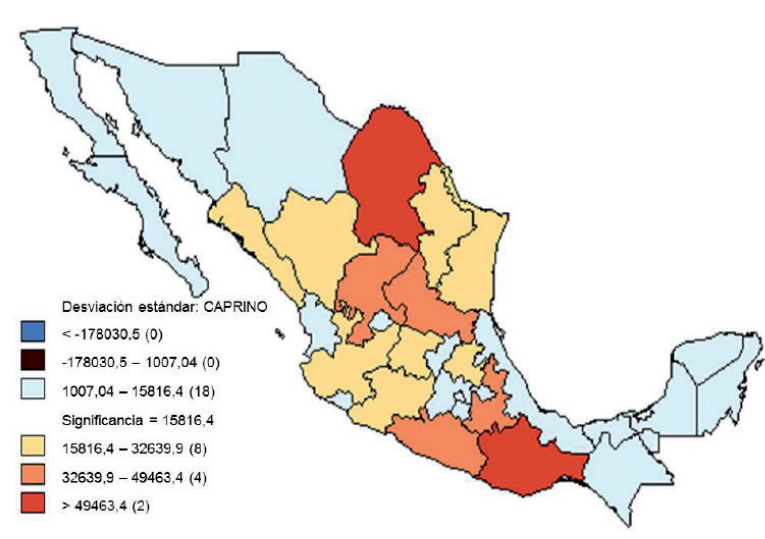

Figura 6. Participación estatal y mapeo de la distribución geográfica de la producción de carne en canal de caprino. México. 2000-2012.

Fuente: elaboración propia con OpenGeoDa ver. 0.9.8.14, a partir de datos de la CNOG (2013).

Figure 6. State participation and mapping of the geographical distribution of goat carcass production 2000-2012, Mexico.

Source: own elaboration with OpenGeoDa view. 0.9.8.14, using data from the CNOG (2013).

producción nacional y como consecuencia desplazar a las de mayor producción.

\section{Autocorrelación espacial}

A través del I se aportó mayor evidencia de la concentración estatal de producción de carne en canal por especie, al mismo tiempo que se identificó la presencia de autocorrelación espacial. Los resultados indicaron que existió correlación espacial positiva baja para la carne de bovino, ovino y caprino, respaldada en los siguientes valores: $\mathrm{I}_{\text {bovino }}=0,0063, \mathrm{I}_{\text {ovino }}=$ $0,1090 \mathrm{y}$ de $\mathrm{I}_{\text {caprino }}=0,2405$; con probabilidades de $0,3111,0,1095$ y 0,0225 , respectivamente (Figura 7). Ante estos valores, en los dos primeros casos se aceptó la hipótesis nula de aleatoriedad, mientras que para la carne de caprino la hipótesis nula se rechazó, mostrando evidencia de que la dependencia espacial positiva fue significativa.

Para la carne de porcino y de pollo los I arrojaron valores negativos: $\mathrm{I}_{\text {porcino }}=-0,1521$ y $\mathrm{I}_{\text {pollo }}=-0,0906$; con probabilidades de 0,3113 y 0,3396 , respectivamente. La correlación negativa en el caso de la carne de pollo fue superior a la carne de porcino, por lo tanto se pudo considerar como una correlación negativa alta. En relación a las probabilidades, en los dos casos se aceptó la hipótesis nula de aleatoriedad, por lo tanto, la dependencia espacial negativa no fue significativa.

La autocorrelación negativa estaría suponiendo que la situación de vecindad de un estado productor, en este caso de carne porcino o pollo, se vería afectado por las condiciones territoriales de sus vecinos, lo que implicaría dificultades para la posibilidad de agrupamientos regionales que ayuden a potenciar los volúmenes de producción, como sugiere la conformación de conglomerados de producción especializada.

Los resultados anteriores mostraron evidencia de la posible conformación de grupos específicos de estados que podrían conformar conglomerados de producción. Particularmente, para la carne de bovino se identificaron dos grupos de estados, que además fueron estadísticamente significativos de acuerdo con la prueba de LISA; el primero integrado por Tamaulipas y San Luis Potosí, y el segundo por Tabasco, Oaxaca y Chiapas (agrupaciones en color rojo en la Figura 8).

La localización geográfica privilegiada de Tamaulipas, sumada a la producción en serie obtenida durante todo el año y el status sanitario e infraestructura acreditados para la exportación, han favorecido el crecimiento anual de su producción en $3,41 \%$, por lo que la implementación de sus sistemas productivos en San Luis Potosí podría impulsar la integración de la cadena productiva, y como consecuencia el crecimiento del sector bovino de carne de esa entidad. Por otro lado, los sistemas de ganadería bovina de doble propósito que imperan en Chiapas, Oaxaca y Tabasco, los podría hacer potencialmente competitivos en la producción de carne a través de la conformación del conglomerados, principalmente a los dos últimos, los cuales conjuntamente aportaron $6,37 \%$ de la producción nacional anual.

Para la carne de cerdo, no hubo evidencia estadística de la posible conformación de un grupo de entidades federativas, que por su volumen de producción pudieran formar conglomerados (Figura 9). Un factor que pudiera explicar estos resultados es el cierre de pequeñas y medianas granjas porcícolas ante la apertura comercial, lo cual generó que la concentración espacial del inventario no correspondiera a las entidades con mayores unidades de producción: $18,8 \%$ del inventario correspondió a Sonora, que solo contó con $0,3 \%$ de las unidades del país; el $11 \%$ a Jalisco, que concentró el 1,6\% de las unidades; 

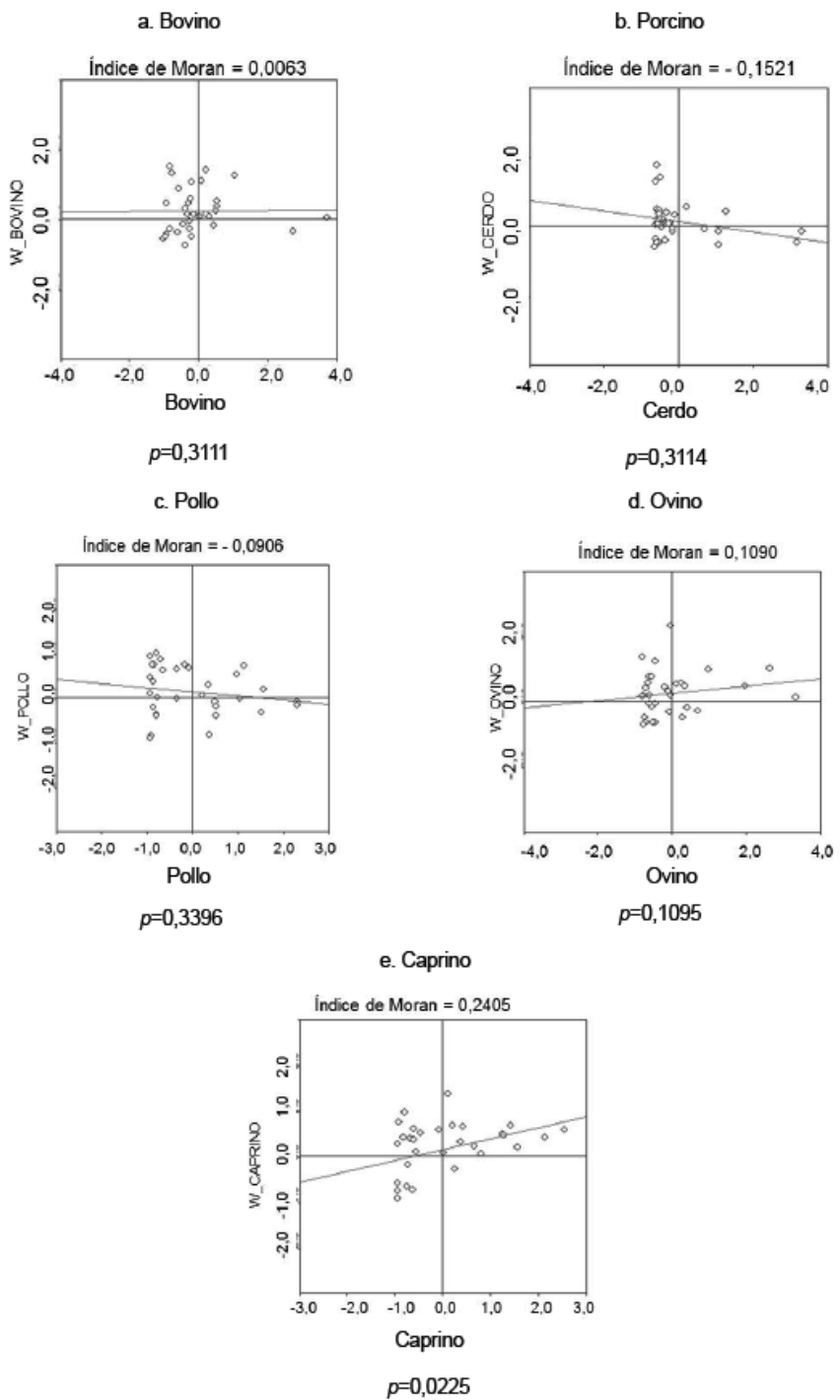

Figura 7. Índices de Moran para los volúmenes de producción de carne en canal por especie, 2000-2012, México.

Fuente: estimación propia con OpenGeoDa ver. 0.9.8.14, a partir de datos de la CNOG, 2013.

Figure 7. Moran's indexes for volumes of meat carcass production by species, 2000-2012, Mexico.

Source: own elaboration with OpenGeoDa view. 0.9.8.14, using data from the CNOG, 2013.

10,7\% a Guanajuato, que participó con el 5,2\% de las unidades; $8,3 \%$ a Puebla, que mantuvo el $11,2 \%$ de las unidades; y el 6,5\% a Veracruz, entidad con mayor participación en las unidades del país $(13,7 \%)$ (Financiera Rural, 2012).
Diez de las 32 entidades federativas, registraron tasas de crecimiento negativas en la producción, registrando una de las peores caídas a nivel nacional en el Distrito Federal, con un decrecimiento anual de (-) $9,08 \%$, esto como consecuencia de la restricción 

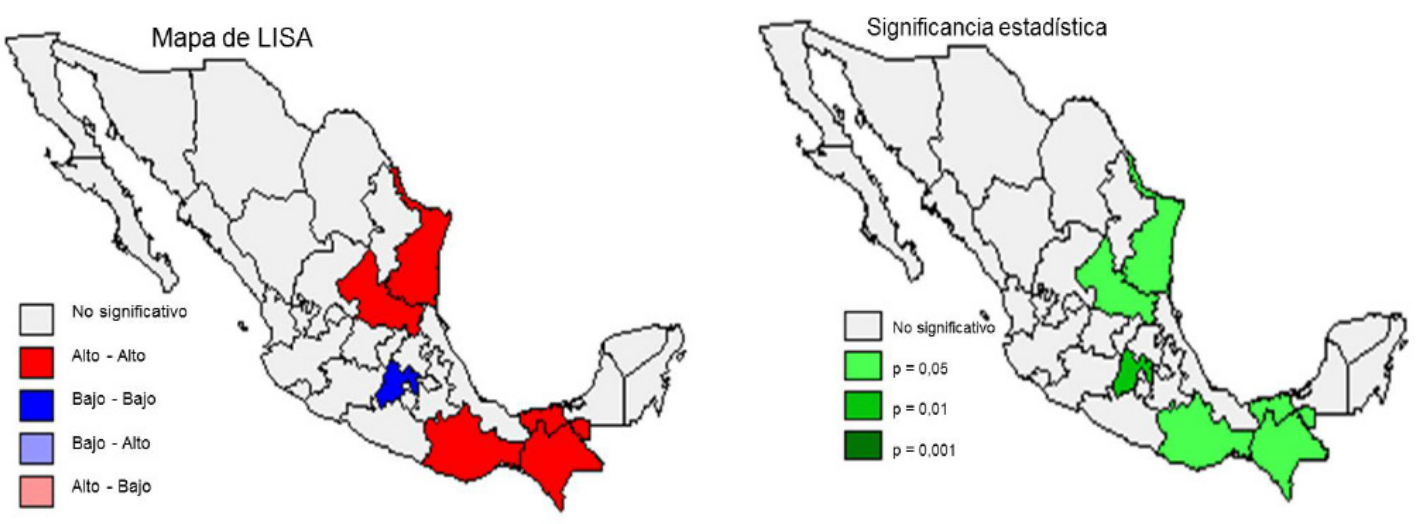

Figura 8. Indicadores de Asociación Espacial Local (Prueba de LISA) para carne en canal de bovino, 2000-2012, México.

Fuente: estimación propia con OpenGeoDa ver. 0.9.8.14, a partir de datos de la CNOG, 2013.

Figure 8. Local Indicators of Spatial Association (LISA test) for cattle carcass 2000-2012, Mexico.

Source: own elaboration with OpenGeoDa view. 0.9.8.14, using data from the CNOG, 2013.
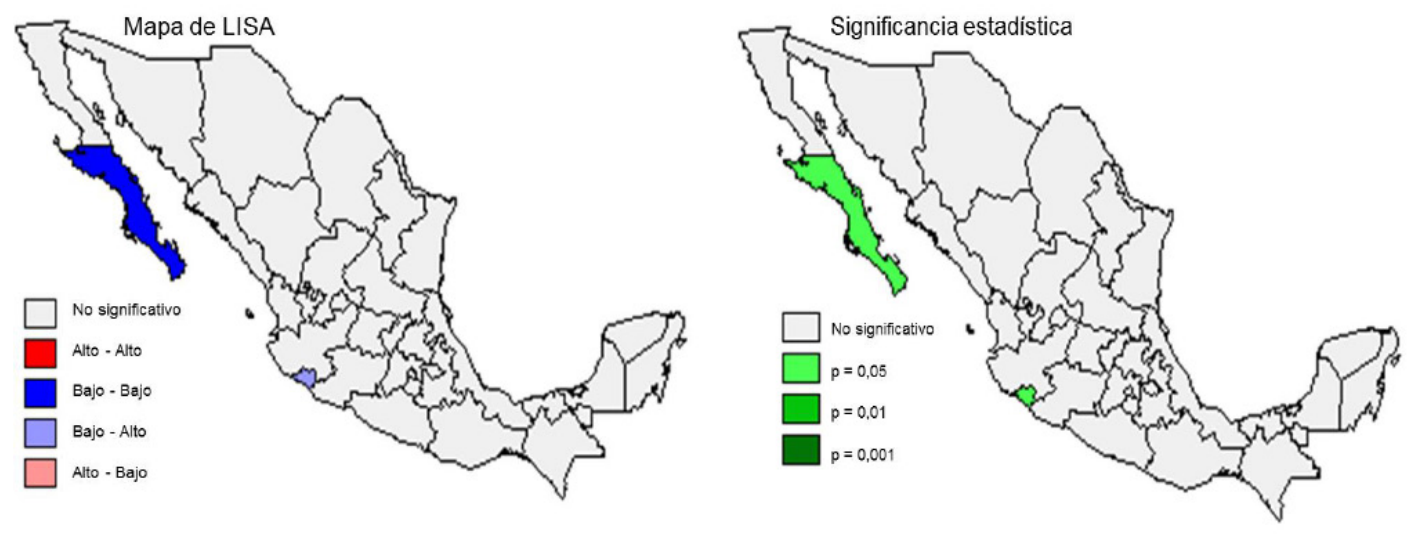

Figura 9. Indicadores de Asociación Espacial Local (Prueba de LISA) para carne en canal de porcino. México. 20002012.

Fuente: estimación propia con OpenGeoDa ver. 0.9.8.14, a partir de datos de la CNOG (2013).

Figure 9. Local Indicators of Spatial Association (LISA test) for pork carcass 2000-2012, Mexico. Source: own elaboration with OpenGeoDa view. 0.9.8.14, using data from the CNOG (2013).

federal para implementar sistemas de producción familiar o de traspatio en esa entidad. Por otro lado, existe la posibilidad que en un futuro Aguascalientes y Colima, pudieran integrarse con Jalisco, dado el crecimiento en su producción, equivalente a 9,83 y $14,66 \%$, respectivamente.

Con significancia del 95\%, para el caso carne de pollo, se identificaron estados, que por sus vecinos, podrían conformar un grupo importante de productores que aprovecharán las ventajas de su localización y de economías de escala para fortalecerse, estos fueron Nayarit, Zacatecas, San Luis Potosí, Hidalgo y Michoacán, los cuales obtuvieron conjuntamente $8,37 \%$ de la producción (Figura 10).

Nayarit incrementó su producción anual en $8,11 \%$, lo cual no le permitió tener una buena participación a 

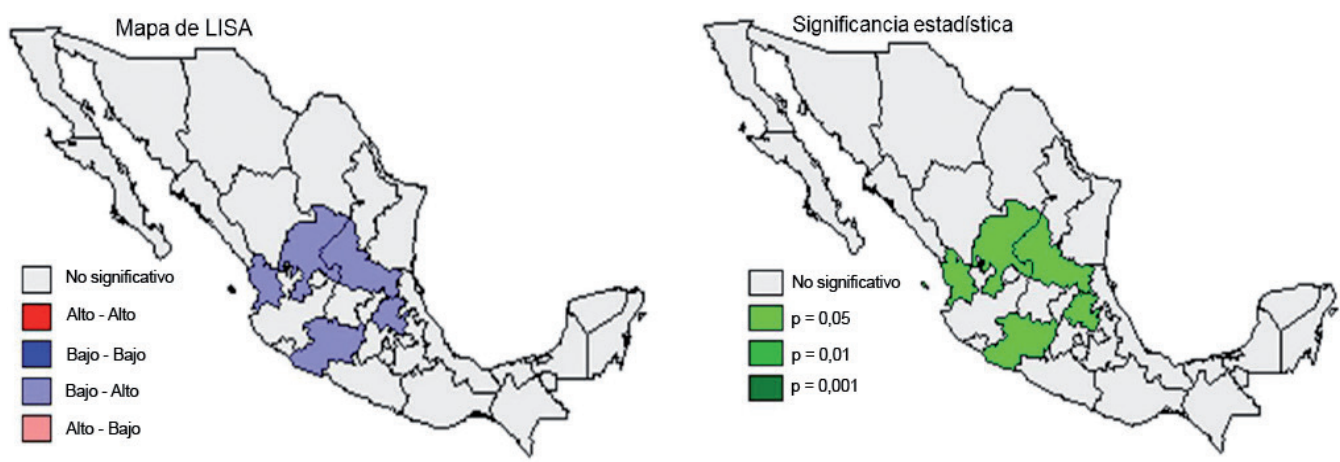

Figura 10. Indicadores de Asociación Espacial Local (Prueba de LISA) para carne en canal de pollo en México. 2000-2012.

Fuente: estimación propia con OpenGeoDa ver. 0.9.8.14, a partir de datos de la CNOG (2013).

Figure 10. Local Indicators of Spatial Association (LISA Test) for chicken carcass 2000-2012, Mexico. Source: own elaboration with OpenGeoDa view. 0.9.8.14, using data from the CNOG (2013).

nivel nacional, dado que únicamente aportó $0,85 \%$ del total; en contraste, Michoacán, el cual a pesar de haber aumentado su producción en únicamente $0,84 \%$, contribuyó con el 1,99\%, lo que significa que existió un mayor inventario de carne de pollo en esta entidad comparado con la primera.

En el caso de la carne de ovino, se identificó un grupo importante conformado por San Luis Potosí,
Hidalgo y Puebla, mismo que se podría consolidar con la integración de Tlaxcala y Querétaro (Figura 11). La consolidación de este grupo podría impulsar la producción en Tlaxcala, debido a que en esa entidad únicamente el 10,9\% de los productores desarrollaron la ovinocultura como actividad primaria; el $85,5 \%$ como secundaria intercalada con la agricultura; y el restante 3,5\% como terciaria (Galaviz et al., 2011). Por otro lado,
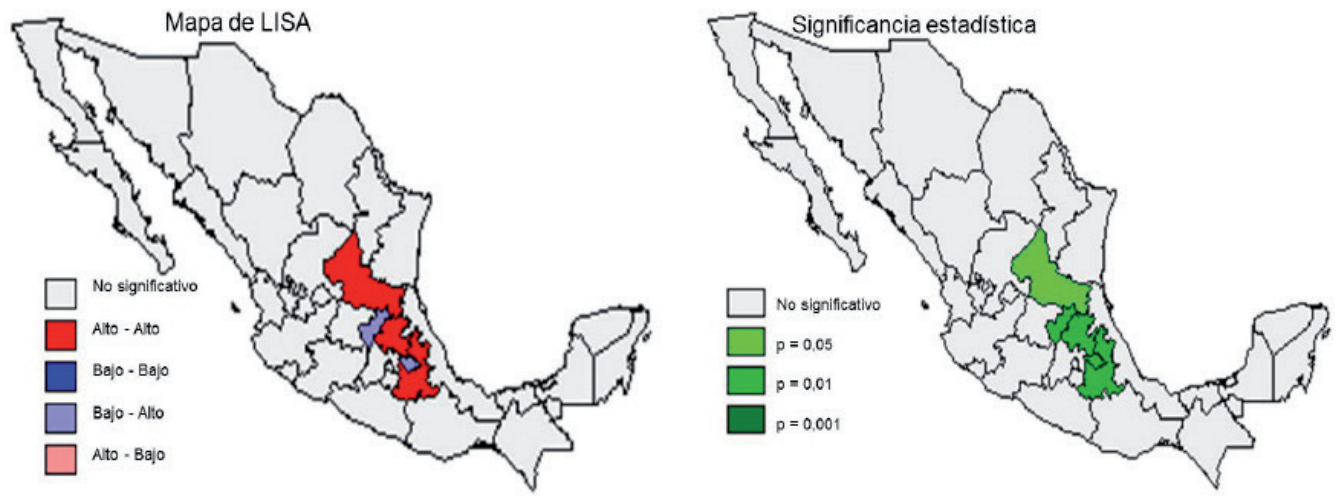

Figura 11. Indicadores de Asociación Espacial Local (Prueba de LISA) para carne en canal de ovino. México. 2000-2012.

Fuente: estimación propia con OpenGeoDa ver. 0.9.8.14, a partir de datos de la CNOG (2013).

Figure 11. Local Indicators of Spatial Association (LISA Test) for sheep carcass 2000-2012, Mexico. Source: own elaboration with OpenGeoDa view. 0.9.8.14, using data from the CNOG (2013). 
se considera que esta integración también fortalecería la capacidad exportadora de Querétaro, ya que a pesar de encontrarse entre los estados más importantes en la zona centro-bajío y contar con una de las mejores genéticas del país, únicamente logró incrementar su producción anual en $2,57 \%$, situación que no le ha permitido cubrir la demanda interna ni la de exportación.

Finalmente, para la carne de caprino se identificaron dos posibles agrupamientos de entidades productoras; el primero en la zona norte integrado por Durango, Zacatecas, San Luis Potosí y Nuevo León; y, el segundo en la Península conformado por Campeche, Yucatán y Quintana Roo (Figura 12). Como se visualiza en el mapa, por la importancia de su producción, Guerrero debería ser el punto central de un nuevo grupo de estados productores; sin embargo, por la vocación productiva de los estados vecinos, no se visualiza su conformación.

En cuanto a la ubicación geográfica, el grupo de la zona norte podría especializarse en la producción de cabrito y cubrir el mercado más importante de este producto que es Monterrey; mientras que, las entidades de la Península, cuya participación en la producción fue casi nula al aportar al año únicamente $0,10 \%$ del total, deberían implementar nuevos sistemas productivos y reestructurar los existentes, a fin de impulsar la actividad, y en el futuro integrar las cadenas productivas.

La desventaja que mostró Guerrero radica en sus sistemas de producción, caracterizados por escasa infraestructura, deficiente manejo de agostaderos y pocas fuentes de comercialización (Fundación Produce Guerrero A.C., 2012); situación similar a la que presentó Oaxaca, dado que la caprinocultura en esa entidad se lleva a cabo bajo sistemas de trashumancia y ramoneo, que generan desequilibrios ecológicos, pero que, dado el papel que juega esta actividad en Huajuapan de León como conservadora de tradiciones culturales, traducida en la elaboración del mole de caderas, y como generadora de empleos directos e indirectos, esta actividad ha prevalecido al punto de ocupar el segundo lugar a nivel nacional (Rodríguez et al., 2011).

El análisis exploratorio de datos y el mapeo de distribución geográfica evidenciaron el grado de concentración espacial de la producción de carne por especie en México obtenida en 2000-2012; a partir del análisis de autocorrelación fue posible identificar la formación de conglomerados estatales para la producción de carne de bovino, caprino y ovino, cuyo patrón de distribución espacial estableció una relación entre las características productivas que favorecen la integración de las cadenas productivas; cumpliéndose así, por un lado con el objetivo de la investigación, y por el otro, la validación de la hipótesis, la cual refiere que existe un efecto generado por la vecindad espacial entre entidades productoras de carne por especie que pueden o no favorecer la conformación de conglomerados entre estas.
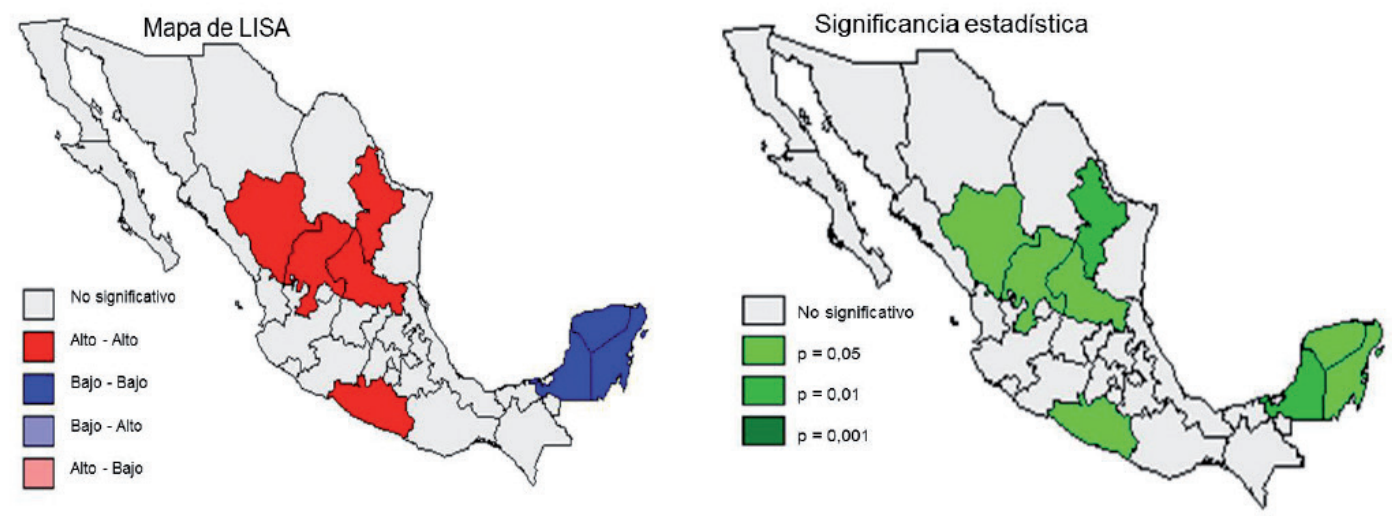

Figura 12. Indicadores de Asociación Espacial Local (Prueba de LISA) para carne en canal de caprino. México. 2000-2012.

Fuente: estimación propia con OpenGeoDa ver. 0.9.8.14, a partir de datos de la CNOG (2013).

Figure 12. Local Indicators of Spatial Association (LISA Test) for goat carcass 2000-2012, Mexico. Source: own elaboration with OpenGeoDa view. 0.9.8.14, using data from the CNOG (2013). 


\section{LITERATURA CITADA}

ANFACA (Asociación Nacional de Fabricantes de Alimentos para Consumo Animal, S.C.). 2013. La producción de alimentos balanceados para consumo animal y el consumo de granos forrajeros en México. En: ANFACA, editores, Memorias económicas, 2011, 2012 y 2013. ANFACA, MÉX.

Anselin, L. 1988. Spacial econometrics: methods and models. Klumer Academic Publishers, London, GBR.

Anselin, A. 1995. Local indicators of spatial associationLISA. Geographical Analysis 27(2):93-115.

CNOG (Confederación Nacional de Organizaciones Ganaderas). 2013. Boletines de información económica pecuaria. Volumen de la producción de carne en canal de bovino, porcino, pollo, ovino y caprino (2000-2011). http://www.cnog.org.mx/index. php?option=com_content $\&$ view $=$ category $\&$ layout $=$ bl og\&id=45\&Itemid=65. (consultado ene. 2013 ).

Fundación Produce Guerrero A.C. 2012. Caprinos, agenda de innovación Guerrero/2012. http:// fundacionproducegro.org.mx/w p-content/ uploads/2012/05/16-Caprinos-Preliminar.pdf. (consulado oct. 2014).

Financiera Rural. 2012. Monografía del ganado porcino. Dirección General Adjunta de Planeación Estratégica y Análisis Sectorial, México, D.F. http://www. financierarural.gob.mx/informacionsectorrural/ Documents/Monografias/Monograf\%C3\%ADaPorcin os\%28jun2012\%29.pdf. (consultado may. 2013).

Galaviz, R., V. López, Z. Ramírez, B. González, R. Bribiesca, G. Rodríguez, y H. Zepeda. 2011. Evaluación territorial de los sistemas de producción en la región nor-poniente de Tlaxcala. Rev. Mex. Cienc. Pecu. 2:53-68.

Gujarati, D. 1995. Basic econometrics. $3^{\text {th }}$ ed. McGrawHill, NY, USA.
Morán, P. 1948. The interpretation of statistical maps. R. Stat. Soc. 10(2):243-255.

Moreno, R., y E. Vayá. 2000. Técnicas econométricas para el tratamiento de datos espaciales: la econometría espacial. Ediciones Universidad de Barcelona, Barcelona, ESP.

Partida, P., B. Varela, J. Severiano, R. Rincón, y B. Rodríguez. 2013. Producción de carne ovina. Libro técnico No. 5. Instituto Nacional de Investigaciones Forestales, Agrícolas y Pecuarias (INIFAP), Ajuchitlán, Colón, Qro., MEX.

Quintana,L.,y G.Mendoza 2008.Econometría básica: modelos y aplicaciones a la economía mexicana. Universidad Nacional Autónoma de México, D.F., MEX.

Rodríguez, G., J.G. Gamboa, J.C. García, y J.G. Rivera. 2011. Tradición sociocultural de la caprinocultura en el estado de Oaxaca: un análisis sobre el potencial económico en beneficio de la región de Huajuapan de León. En: B.A. Cavalloti et al., editores. La ganadería ante el agotamiento de los paradigmas dominantes. Vol. 2. Universidad Autónoma de Chapingo, Texcoco. MEX. p. 321-334.

SIACON (Sistema de Información Agroalimentaria de Consulta). 2013. Programa informático con base de datos agrícolas, pecuarios y pesqueros. Volumen de la producción de carne en canal de bovino, porcino, pollo, ovino y caprino (2000-2011). http://www.siap. gob.mx/optestadisticasiacon2012parcialsiacon-zip/ (consultado abr. 2013).

SENASICA (Servicio Nacional de Sanidad, Inocuidad y Calidad Agroalimentaria). 2012. Padrón estatal de rastros. http://www.senasica.gob.mx/?id=4846 (consultado mar. 2013). 
